\title{
Archipel
}

ARCHIPEL Études interdisciplinaires sur le monde insulindien

93 | 2017

Varia

\section{Note sur le Musée-bibliothèque des Chinois peranakan de Tangerang, Jakarta}

SONG Ge

\section{(2) OpenEdition}

Journals

Édition électronique

URL : http://journals.openedition.org/archipel/418

DOI : 10.4000/archipel.418

ISSN : 2104-3655

Éditeur

Association Archipel

Édition imprimée

Date de publication : 6 juin 2017

Pagination : 219-228

ISBN : 978-2-910513-74-0

ISSN : 0044-8613

Référence électronique

SONG Ge, " Note sur le Musée-bibliothèque des Chinois peranakan de Tangerang, Jakarta », Archipel [En ligne], 93 | 2017, mis en ligne le 01 juin 2017, consulté le 02 octobre 2020. URL : http://

journals.openedition.org/archipel/418; DOI : https://doi.org/10.4000/archipel.418 
SONG $G_{E}^{*}$

Note sur le Musée-bibliothèque des Chinois peranakan de Tangerang, Jakarta

\section{Historique}

Le Museum Pustaka Tionghoa Peranakan, est le premier du genre à être consacré aux écrits émanant de la communauté sino-indonésienne. Il a été fondé dans le district de Serpong, Tangerang Sud, en 2012, à l'initiative de Monsieur Azmi Abu Balear, ingénieur de formation âgé d'une quarantaine d'années, reconverti dans la vente de livres d'occasion. Avec son épouse, Madame Fajrieni, il possède et gère deux librairies, dont l'une en face du musée-bibliothèque, et l'autre à Bekasi.

Il y a déjà une dizaine d'années que Monsieur Azmi a commencé à collectionner des ouvrages se rapportant à la littérature et à l'histoire des peranakan. En 2012, il loua un bâtiment à deux étages pour fonder son muséebibliothèque. Cependant, il fut obligé de le fermer temporairement au milieu de l'année 2014, la propriétaire ne souhaitant plus louer son bien comme musée, de peur d'avoir des ennuis avec l'administration. En conséquence, Monsieur Azmi décida d'acheter une maison, toujours dans le district de Serpong, pour abriter sa collection. Après divers préparatifs qui durèrent près d'un an, le musée-bibliothèque rouvrit ses portes en octobre 2015. À présent, il comprendrait au minimum quelque 20000 volumes, auxquels il faut encore ajouter des journaux, des ouvrages divers ainsi que des documents et des objets personnels.

*. INALCO, Paris 
Le nouveau siège du musée-bibliothèque est dans une rue commerçante, néanmoins très calme, dont les bâtiments assez uniformes et colorés rappellent par leur style certaines architectures du Moyen-Orient. L'édifice, dont l'extérieur est peint en blanc, a une superficie d'environ $120 \mathrm{~m}^{2}$ répartie sur deux étages. Monsieur Azmi l'a aménagé avec grand soin : le panneau au-dessus de la porte d'entrée porte le nom de l'institution en caractères dorés délicatement gravés sur un fond rouge vif; les étagères et les présentoirs de livres sont également des vintages du mobilier peranakan; l'objet le plus apprécié par Monsieur Azmi, et qui attire les regards dès l'entrée, est un ancien panneau portant le nom de 中华会馆 (Tiong Hoa Hwee Kwan, THHK) ou Association chinoise ${ }^{1}$. Cette association fut d'abord créée à Batavia en 1900 afin de prendre la défense de la nation (bangsa) chinoise dans son ensemble et de créer des écoles. Elle essaima ensuite dans les autres villes de Java et des îles extérieures. À noter que le musée-bibliothèque expose également une calligraphie (anonyme) encadrée qui se lit Tianxia wei gong 天下为公 ou « le monde est un bien commun », laquelle rappelle ici l'idéal politique de Sun Yat-sen, le fondateur de la République de Chine.

Lors de notre première rencontre avec Monsieur Azmi et son épouse, une de nos questions fut de savoir pourquoi ils se sont intéressés à la culture des peranakan, puisqu'ils sont originaires respectivement d'Aceh et de Minangkabau et que, par ailleurs, ils ne connaissaient pas beaucoup la littérature sino-malaise. D'après Madame Fajrieni, au début ils collectionnaient ce genre de livres en raison de leur rareté. À une époque où tous les écrits se rapportant à la Chine étaient interdits en Indonésie, ces ouvrages leur semblaient " audacieux, voire subversifs ». Après s'être documentés, ils décidèrent de faire quelque chose pour les peranakan qui, d'après eux, ont beaucoup contribué à l'édification de l'Indonésie mais ont été longtemps traités de manière injuste. « Nous souhaitons établir un musée-bibliothèque qui appartienne à tous les peranakan ainsi qu'aux personnes intéressées par leur littérature », a dit Madame Fajrieni.

C'est sans aucun doute une décision assez courageuse. Jusqu'aujourd'hui, en effet, les écrits sur les peranakan, encore considérés comme sensibles, sont surveillés par les autorités indonésiennes. Avant l'ouverture du muséebibliothèque, Monsieur Azmi a été convoqué par les autorités municipales pour exprimer sa motivation. Madame Fajrieni nous a expliqué que si c'était un peranakan qui avait voulu créer une telle institution, cela lui aurait été fort difficile, voire impossible. Après l'établissement du Museum Pustaka, beaucoup de peranakan sont venus exprimer leur gratitude et leur soutien à Monsieur Azmi.

1. Monsieur Azmi l'a acheté à Semarang, mais d'après la transcription hokkien du toponyme, il s'agirait plutôt du panneau de la branche de la THHK de Sukaraja (Java ouest). 


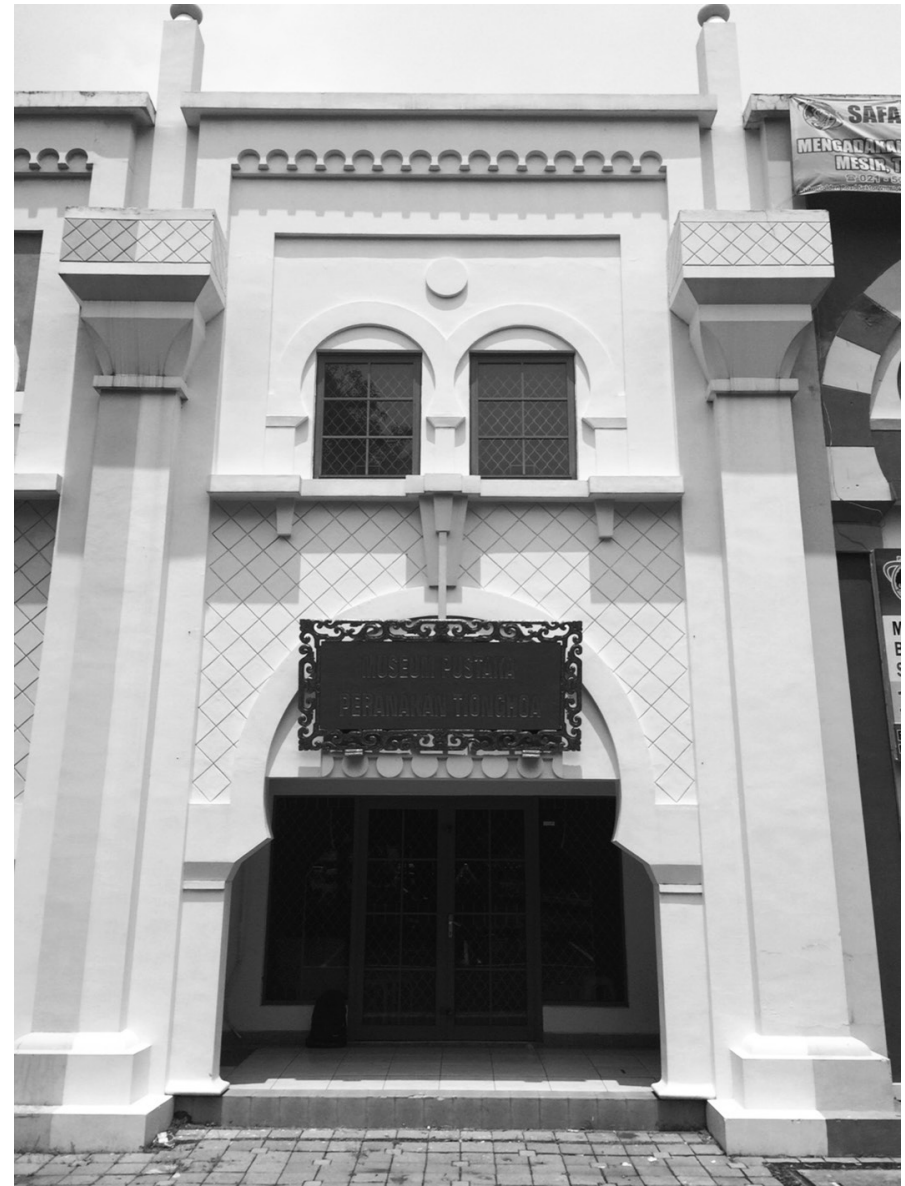

Fig. 1 - Façade du Museum Pustaka Peranakan Tionghoa. (Cliché : Song Ge, 2016)

Les sources de cette collection sont de deux sortes. Il y a à la fois des ouvrages qui ont été donnés par leurs propriétaires ou leurs descendants, et d'autres qui ont été acquis auprès de familles qui n'en comprenaient plus l'intérêt et voulaient s'en défaire. Lors de telles ventes privées, que ce soit à Java, à Sumatra ou même dans la péninsule malaise, Monsieur Azmi est souvent averti en premier. Il n'est pas difficile d'imaginer qu'après quelque quarante années durant lesquelles ces livres ont été conservés à grand-peine, les goûts littéraires des peranakan aient changé. Il y a une rupture culturelle très nette entre les générations, et les jeunes ne sont plus à même de percevoir l'intérêt de ces ouvrages. 


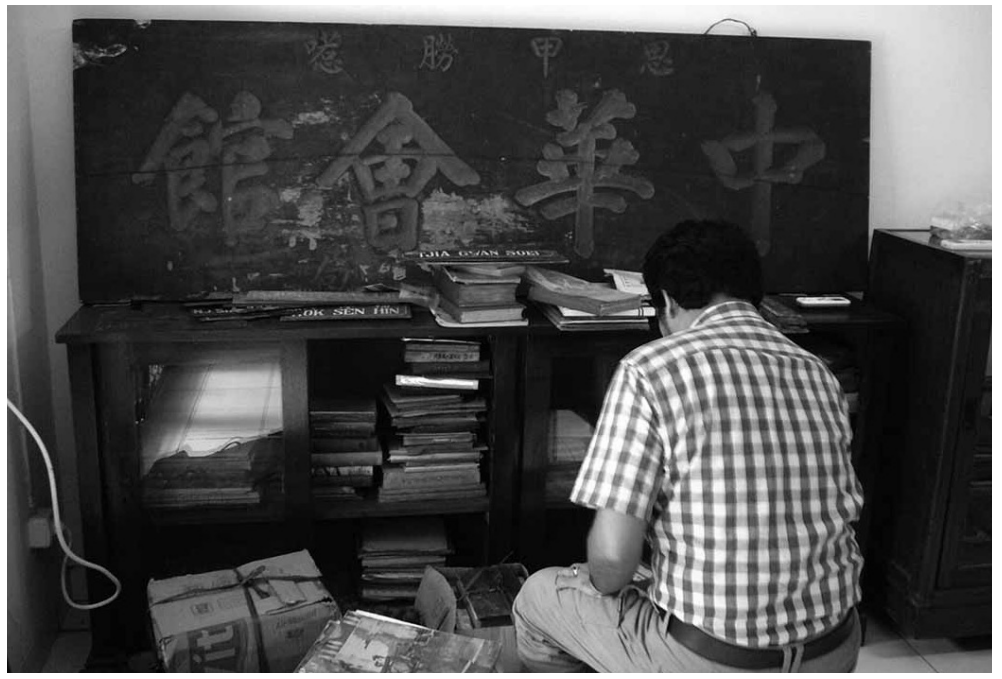

Fig. 2 - Monsieur Azmi travaille dans le musée-bibliothèque, devant un ancien panneau de la Tiong Hoa Hwee Kwan. (Cliché : Song Ge, 2016)

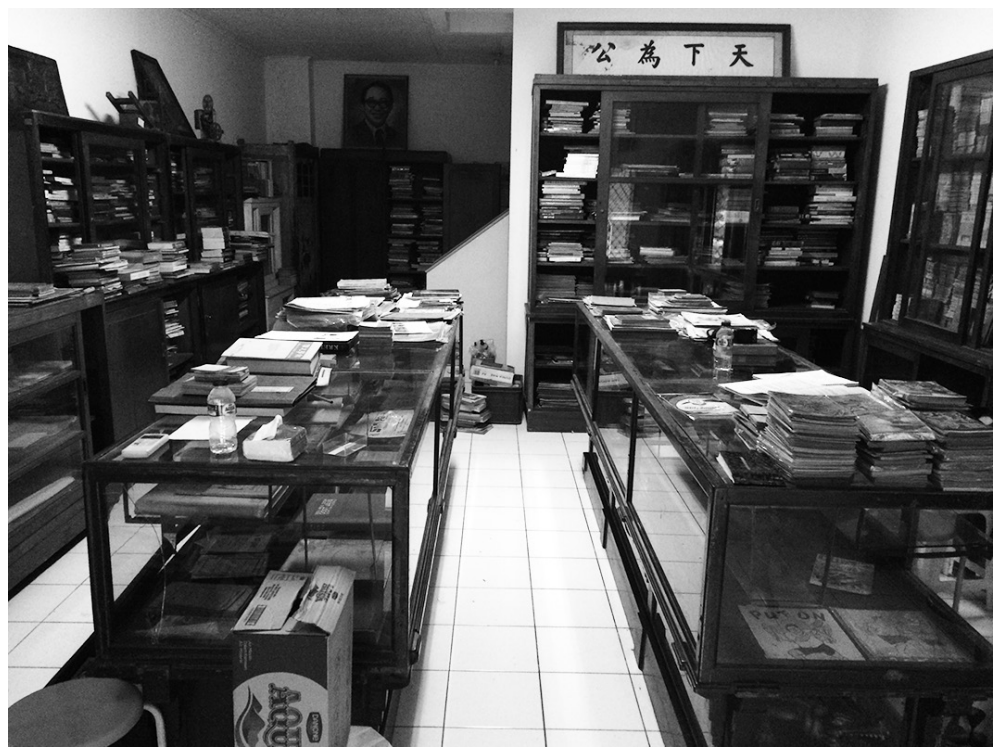

Fig. 3 - Rez-de-chaussée du Museum Pustaka Peranakan Tionghoa. (Cliché : Song Ge, 2016) 


\section{Coup d'œil sur les collections}

Les collections ainsi rassemblées sont impressionnantes, tant par leur quantité que par leur qualité. Faute de temps et de moyens, celles-ci ne sont pas encore bien rangées et classifiées, d'autant plus qu'après le déménagement du musée-bibliothèque, un certain nombre de livres sont restés dans les cartons. Cependant, après avoir effectué des recherches préliminaires dans ledit Museum Pustaka en mars 2016, il nous est apparu que ces collections sont très riches. Elles peuvent se répartir en cinq catégories :

\section{Euvres littéraires}

Les collections les plus anciennes du musée sont des traductions de romans chinois datant des années 1890, telles Pek Koei Tjie 白圭志 《Histoire du sceptre blanc» et Pak Ijoe 北游记 «Récit du pèlerinage vers le Nord». Selon notre enquête, il y a au moins trente-trois traductions (rééditions exclues) de romans chinois traditionnels, tels que Sam Kok三国(演义) 《Roman des Trois Royaumes », Tjoen Tjioe Ngo Pa春秋五霸 《Les cinq despotes de l'époque des printemps et des automnes », Tong Tjioe Liat Kok东周列国 《Royaumes des Zhou de l'Est », See Yoe西游(记) « Récit de pèlerinage vers l'Ouest », Lek Bowtan 绿牡丹 《La pivoine verte》 et Hong Sien 封神(演义) «Récit de l'investiture des dieux ». La plupart furent publiées au début du $\mathrm{Xx}^{\mathrm{e}}$ siècle, en petits fascicules afin de favoriser les acheteurs peu argentés. Ces livres sont relativement abîmés, signe qu'ils ont été beaucoup lus.

Au premier abord, il semblerait que les œuvres originales en malais des Sinoindonésiens soient moins bien représentées que les traductions de romans chinois. Toutefois nous avons repéré quelques syair ${ }^{2}$, ainsi que des romans parus entre les années 1910 et 1960. Certains publiés sous forme indépendante, d'autres dans des revues littéraires. Parmi les premiers, citons Tambahsia, soewatoe tjerita jang betoel soedah kedjadian di Betawi antara tahoen 1851-1856 ou « Tambahsia, une histoire vraie qui a eu lieu à Batavia pendant les années 1851-1856 » (3 éd., 1914) ; Tan Boen Kim, Nona Kim Lian, Soeatoe tjerita jang betoel telah terdjadi di kota Canton sabelonnja petja revolutie jang kasatoe di Tiongkok ou « Nona Kim Lian, une histoire qui s'est passée à Canton au seuil de la première révolution chinoise » (Batavia, 1916); citons enfin Tjoe Hong Boe, Setangan berloemoer darah « un mouchoir taché de sang 》 (Semarang, 1927). Quant aux autres romans, ils paraissaient dans les revues littéraires, telles que Tjerita Roman et Tjilik Roman's, contemporaines de celles publiant des traductions de romans de cape et d'épée dont nous allons maintenant parler.

2. Par exemple, celui de Djali-Djali, Sair-tjerita Nona Glatik, Satoe gadis jang berparas eilok dan tersohor tjantik, Potongan ramping, pinggangnja langsing dan lentik, Ibarat boenga, banjak orang jang ingin petik, Batavia: Tan Thian Soe, 1921. Le syair est une poésie malaise, de longueur indéterminée, et composée de strophes de quatre vers de quatre mots de rimes identiques. 


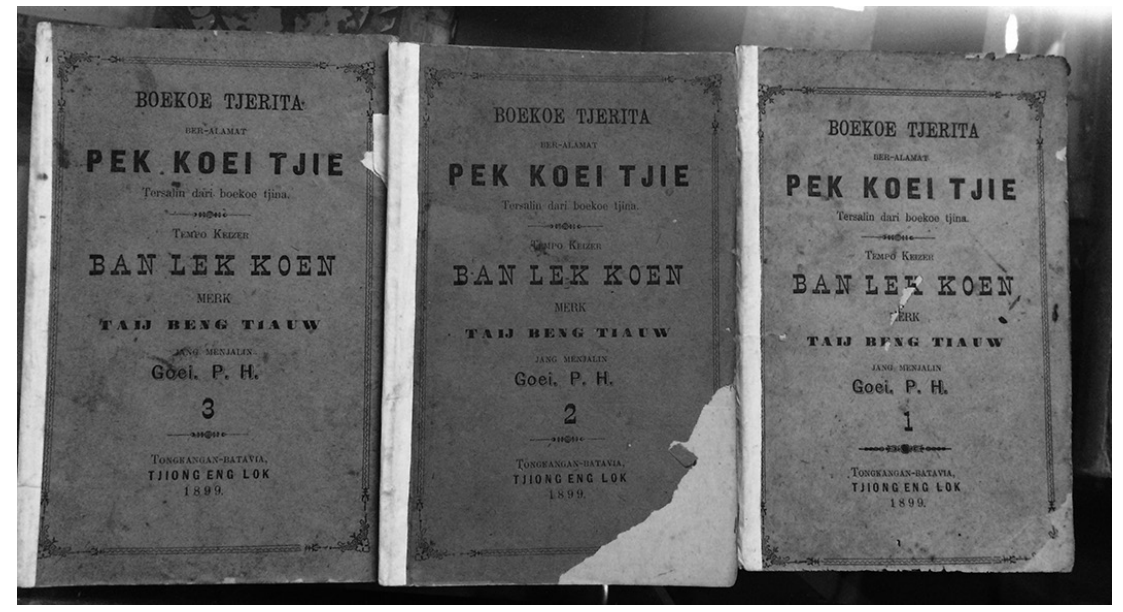

Fig. 4 - Couvertures de la traduction du Pek Koei Tjie 白圭志 de 1899. (Cliché : Song Ge, 2016)

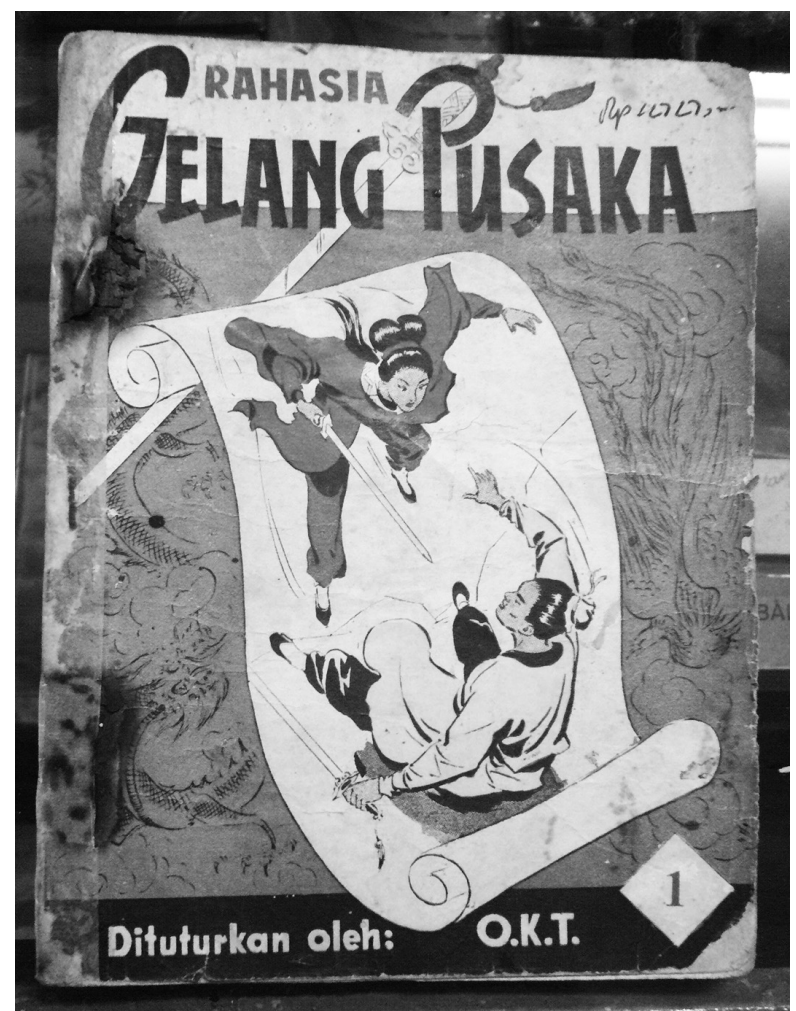

Fig. 5 - Couverture du roman de cape et d'épée Rahasia Gelang Pusaka « Secret du bracelet de famille », traduit par O. K. T. (Cliché : Song Ge, 2016) 


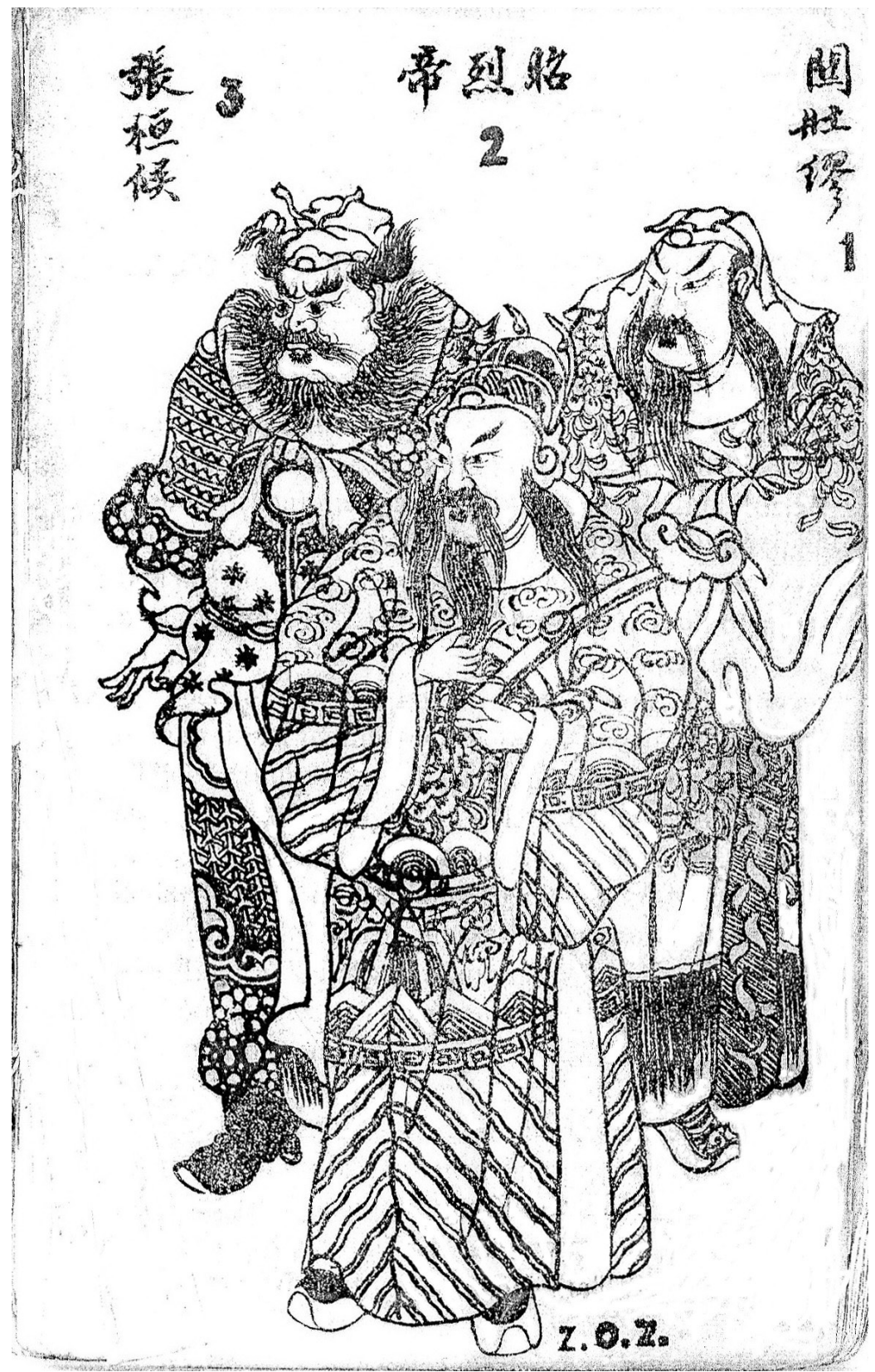

Fig. 6 - Illustration tirée du Sam Kok de Tjie Tjin Koeij en 62 vol. (1910-13), représentant les trois principaux héros du roman : de droite à gauche, Guan Yu, Liu Bei, et Zhang Fei. 
Les cerita silat, romans de cape et d'épée d'origine chinoise, ou wuxia xiaoshuo 武侠小说, sont également bien représentés dans le musée. Il faut mentionner que Ong Kim Tiat 王金铁 (1893-1964) et Oey Kim Tiang 黄金长 (1903-1995), deux traducteurs peranakan célèbres et prolifiques, habitaient Tangerang. Parmi les cinquante-quatre romans de cape et d'épée que nous avons recensés, trente sont signés des initiales O. K. T. De fait, ces deux traducteurs utilisaient les mêmes initiales. Ong Kim Tiat, qui fut le maître de Oey Kim Tiang à l'école de la Tiong Hoa Hwe Koan de Tangerang, est resté moins célèbre que son élève. Jusqu'à présent, on n'arrive pas encore bien à attribuer à chacun de ces deux traducteurs les œuvres qui leur reviennent. Un autre problème est celui de la datation de ces traductions. En effet, elles ne comportent pas de date de publication, sauf deux, parues respectivement en 1961 et en 1964. Si on se base sur le style des couvertures et l'état des livres, on peut penser que ces traductions ont probablement été publiées dans les années 1950-1960, car toutes sont écrites selon l'orthographie antérieure à la réforme de 1972.

Parmi les revues littéraires consacrées aux traductions de roman de cape et d'épée, très à la mode en Indonésie depuis les années 1920, les trois suivantes sont très bien représentées : plus de 270 numéros de Gie Hiap 义侠, « Le héros altruiste », 362 de Boe Hiap 武侠 « Le combattant », ainsi qu'une collection complète de Lishi xiaoshuo 历史小说 《Romans historiques » (1952-1955).

\section{Journaux et revues}

Vu que les peranakan ont joué un rôle important dans le développement de la presse en Indonésie, leurs journaux et leurs revues font partie intégrante des collections. Parmi ceux conservés dans le musée, le Sin Po, journal alors le plus influent, est le mieux représenté. Ses premières éditions, $\mathrm{n}^{\circ} 1-10$ en 1910 et $\mathrm{n}^{\text {os }}$ 49-50 en 1911, très rares, ont été données par Madame Myra Sidharta. Figurent également quelques exemplaires pour l'année 1949. Il y a aussi une cinquantaine de numéros de l'édition hebdomadaire, Sin Po wekelijkscheeditie (fondée en 1923), pour l'année 1926. Nous avons repéré également certains numéros des périodiques suivants : Ik Po 译报 (1904), hebdomadaire progressiste édité à Surakarta par Tjoa Tjoe Koan 蔡珠贯 (1861-1905), Keng Po 竞报 (1949), Berita Indonesia (1948), Star Weekly (1953), ces trois derniers publiés à Jakarta ; enfin, Kuang Po (1957) ${ }^{3}$ et $\operatorname{Sin}$ Min 新民 (1968), tous deux imprimés à Semarang.

3. Kuang Po est devenu Suara Indonesia; cf. Benny G. Setiono, Tionghoa dalam Pusaran Politik, Jakarta: Elkasa, 2003, p. 465. 


\section{Ėtudes diverses se rapportant aux Chinois et aux Indes orientales néerlandaises}

Le musée-bibliothèque a également un riche fonds d'études (en langues diverses) se rapportant à la littérature, l'économie, la culture ainsi qu'à l'histoire des peranakan et des Chinois. Les travaux spécialisés sur la littérature des peranakan, comme Literature in Malay by the Chinese of Indonesia, a provisional annotated bibliography de Claudine Salmon, sont placés en bonne position dans le musée. À part les recherches récentes, nous avons aussi découvert des études plus anciennes et difficiles à trouver de nos jours, telle la première édition du Lan ling dongyindu shi 兰领东印度史 / Geschiedenis van Nederlandsch Oost Indië " Histoire des Indes orientales néerlandaises », rédigée par P.G. Siem (Shen Tieya 沈铁崖), et publiée à Shanghai par la Shanghai Commercial Press 上海商 务印书馆en 1924. Elle comporte une préface, en néerlandais, du fonctionnaire honoraire chargé des affaires chinoises à Semarang, A.G. de Bruin, et une autre en chinois de l'auteur.

\section{Ouvrages historiques, didactiques, et religieux}

Il s'agit de publications diverses, en malais et en chinois, ne concernant pas la littérature au sens propre, mais qui sont précieuses en tant que sources historiques. Tels par exemple des livres commémoratifs de la T.H.H.K., dont celui de Nio joe $\mathrm{Lan}^{4}$, des albums souvenirs d'écoles chinoises, des dictionnaires chinois-malais, des livres sur la médecine traditionnelle chinoise, sur la cuisine peranakan et le fengshui. Ils comprennent également des textes religieux en chinois, tels Yulan shenghui baojuan 孟兰盛会宝卷 《Rouleau précieux ${ }^{5}$ pour la grande réunion en vue du soulagement des âmes des défunts 》, Cibei Lianghuang baochan 慈悲梁皇宝忓 《Précieux texte de repentir de l'Empereur Liang pour aider les âmes en détresse (à obtenir la rémission de leurs péchés) 》, 关帝明圣经注 “Commentaire du Canon de l'illustre et sage empereur Guan » (1939). Ces derniers textes nous permettent de mieux comprendre les croyances populaires au sein des communautés chinoises en Asie du Sud-Est de la fin du XIX et du début du XX⿳亠丷厂 ${ }^{\mathrm{e}}$ siècles.

\section{Documents et objets personnels}

Cette dernière catégorie comprend à la fois des documents de famille dont des portraits, des spécimens de pièces d'identité (cartes de résidence, permis de voyage pour les peranakan, passeports), et même des bulletins scolaires provenant d'écoles chinoises. À noter aussi divers objets, notamment une plaque sur laquelle le nom chinois du propriétaire d'une maison est noté en caractères latins d'un côté, et de l'autre, celui en indonésien, ce qui montre

4. Nio Joe Lan, Riwajat 40 taon dari Tiong Hoa Hwe Koan Batavia, 1900-1939, Batavia, sans maison d'édition, 1940.

5. Les baojuan constituaient un genre littéraire consacré à l'édification religieuse. 
qu'elle avait été réemployée lors de la politique incitant fortement les personnes d'origine chinoise à changer de nom.

En bref, ce fonds se démarque de ceux des bibliothèques publiques d'Indonésie par le fait qu'il comporte de précieux ouvrages en chinois se rapportant à la culture traditionnelle, une petite mais intéressante collection de littérature grise, ainsi qu'un certain nombre d'études anciennes sur la communauté peranakan d'Indonésie, rédigées en des langues diverses. Autre particularité, sa très riche collection de traductions de romans chinois et de cerita silat.

\section{État des lieux}

Concernant le développement futur du Museum Pustaka, Monsieur Azmi nous a dit vouloir continuer à collecter le plus d'ouvrages possible. Néanmoins, il y a également un urgent besoin d'aide professionnelle pour amorcer le plus rapidement possible le classement et l'enregistrement des collections, et ce, afin de rendre le musée-bibliothèque le plus opérationnel possible.

Il demeure que tous les efforts faits par Monsieur Azmi et son épouse sont très touchants et encourageants pour nous. Durant nos séances de travail, nous avons rencontré deux hommes peranakan d'une cinquantaine d'années. L'un d'eux était venu pour donner une biographie succincte de son père, un ancien propriétaire terrien de Bekasi qui passait pour avoir été un bienfaiteur, mais qui, néanmoins, avait perdu tous ses biens dans les années 1950. Pour ce monsieur, déposer la biographie de son père dans le musée-bibliothèque est une façon de garder vivant le souvenir de son père.

Il nous est apparu que le Museum Pustaka ne se limite pas seulement à rassembler des collections ; c'est aussi un point d'ancrage historique, un espace privilégié où les peranakan peuvent rafraîchir leurs souvenirs, voire leur sentiment identitaire, et peut-être qu'un jour, il attirera aussi de jeunes peranakan désireux de se pencher sur l'histoire de leurs ancêtres ${ }^{6}$.

\section{Coordonnées du Museum Pustaka Tionghoa Peranakan:}

Le musée est actuellement accessible à tout un chacun, mais exclusivement sur rendez-vous, à prendre soit par téléphone, soit par courriel.

Adresse : Ruko Golden Road C28/25

CBD BSD

Jl. Pahlawan Gudang, Serpong,

BSD City

Tangerang Selatan, 15320

Email : museumpustakationghoaperanakan@gmail.com

Téléphone: 082124925243

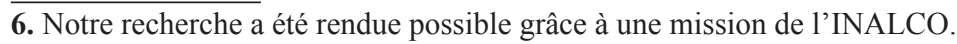

